\title{
Civilistic approach to the concept of robot as an object of civil law
}

\author{
Vera Borshcheniuk $^{1}$, Nina Semeryanova ${ }^{1}$, Uliana Filatova $^{2}$, Valeriy Zhabskiy ${ }^{3 *}$ \\ ${ }^{1}$ South Ural State University (National Research University), 628600, 9, Mira str., Chelyabinsk, \\ Russia \\ ${ }^{2}$ Irkutsk State Universities, 664003, 1, Karl Marx str., Irkutsk, Russia \\ ${ }^{3}$ Moscow University of the Ministry of Internal Affairs of the Russian Federation named after V.Y. \\ Kikot, 117437, 12, Academician Volgin str., Moscow, Russia
}

\begin{abstract}
The relevance of the study is specified by the issue of determining the status of robots in civil law. The paper attempts to establish the legal identification of robot as an object of civil rightsin order to enshrine in the law provisions on this new object. Classification of robots is also proposed, which can serve as the basis for subsequent systematization of robots and their registration systems. The leading research approaches used by the authors are such scientific methods as dialectics, analysis, synthesis, deduction, comparative legal and formal legal method. Conclusions: definition of digital and virtual robot is proposed. The authors believe that digital robot does not have to possess signs of anthropomorphism, the main thing in it is the level of intelligence. Anthropomorphism should currently be considered from the point of view of resembling a person by intellect, not by physical characteristics.
\end{abstract}

\section{Introduction}

Research in the field of artificial intelligence is currently very popular among scholars of various branches of law, since "smart" technologies are actively introduced in most areas of public relations. Their wide distribution is caused by the automation of production, the need for processing and analysis of a huge amount of information and the continuous development of scientific and technological progress.

The attitude to artificial intelligence in the scientific community is not unambiguous. The term "artificial intelligence" is practically not used. As a rule, more specific terms are used, such as an intelligent system, machine learning, evolutionary algorithms, and many others. At the same time, there are many definitions of the term "artificial intelligence". In particular, artificial intelligence is understood as a section of computer science devoted to modeling human intellectual activity. More generally, this is a copy of the mapping of neurons in the brain. Although this scientific approach is subject to constructive criticism. Thus, a number of authors believe it is not artificial intelligence that is capable of evolving, but the subject of artificial intelligence. In this connection, it is necessary to look for the

*Corresponding author: a.copytowa@yandex.ru 
ways of evolutionary development of artificial intelligence, since the revolutionary way of its creation, which most often is offered in form of a simple copy of the neural structure of the brain, seems unrealistic as well as methodologically erroneous [1].

In relation to the topic of research, artificial intelligence can be defined as the ability of a digital computer or a computer-controlled robot to perform tasks usually associated with intelligent creatures. Artificial intelligence technologies are actively used in robotics, which is then introduced into civilian circulation. A new specific object of social relations (robot) appears in the legal field. In this connection, there is a need to establish its legal regime.

Theoretical basis of the study is based on the scientific research of such scientists as N.M. Abdikeev, A.N. Averkin, R.A. Aliev, P. Norvig, G.S. Osipov M.G. Gaze Rappoport, S. Russell, D.A. Pospelov, M.M. Shakhnazarov and others.

In the legal literature, the issue of legal nature of robots is debatable. Traditionally, robot is considered as an object of law, in some cases it is considered as a subject. Is there a need to give a robot legal personality? It ia a question that needs to be resolved. So, according to E. Blavatsky, "Truth will never come down to us. We must rise to it." Therefore, conducting comprehensive studies and developing unified systematic approach to regulating relations, which onset is inevitable, will help to avoid future adverse consequences of inefficient regulation.

The establishment of legal identification of robots is advisable to begin with the definition of "what is a robot" and what classifications of robots are currently used.

The term "robot" is of Czech origin (robota means forced labor). According to the explanatory dictionary of S.I. Ozhegov robot is an automaton that performs actions similar to human actions [2]. Collier's Encyclopedia defines a robot as an automatic device designed to carry out production and other operations usually performed by humans. In technical literature, robot is spoken of as an electronic-mechanical device capable of expedient behavior in a changing external environment and performing work operations with complex spatial movements [3]. The international standard ISO 8373: 2012 defines robot as an actuator programmable in two or more degrees of mobility, that has a certain degree of autonomy and is able to move in external environment in order to perform its intended tasks.

The term "robot," does not have enduring certainty. It is considered as an automatic machine (chat robot), as an electronic-mechanical device (unmanned aerial vehicle), as an electromechanical, pneumatic and hydraulic device (robot car), or as a robot program (bot). In general, robot is understood as a technical device with properties of certain behavior and, as a rule, spatial movements.

Certain classification of robots was developed in the scientific literature: a) by purpose: industrial (up to $80 \%$ of the total fleet of robots in the world) and service (robot that does useful work for people and equipment, excluding industrial automation tasks); b) by the type of operations performed: technological and auxiliary, which are designed to perform auxiliary technological operations for servicing the main technological equipment (in this regard, technological robots are referred to the main technological equipment, and auxiliary robots are referred to automation); c) the breadth of the list of operations the robot is intended to perform: special ones designed to perform one specific technological operation (for example, welding, coating, specific assembly operation), specialized ones that can perform several operations of the same type (for example, an assembly robot with interchangeable working tools) and universal, performing various basic and auxiliary operations within their technical capabilities; d) according to the method of placement: floor, suspended, built into other equipment; d) according to the control method: with software, adaptive and intelligent control.

Definition of a robot in terms of its functionality seems interesting. So, robot can be called anything performing its intended actions, a device that simultaneously meets three 
conditions: it is able to perceive the world around with the help of sensors; able to understand the surrounding physical world and build patterns of behavior (program, neuron) to perform work in a functional environment; affect the physical world in one way or another.

The classification of robots considered above is classical; it is used in formation of type of robots and their names.

\section{Discussion}

In the context of digitalization of economy and technological breakthroughs, robots based on digital technologies are of great interest. The purpose of such developments is to create a robot with the properties inherent in living things. We are talking only about nutrition, movement and development. The level of intellectual abilities of robots increases. It is planned to achieve this goal with help of digital technologies. Opinions of scientists involved in research in the field of robotics on this issue are diverse. Some believe that digital technologies have a number of negative features, in particular, robot on analog circuits is more adapted to the environment than digital one. Its effectiveness ends when it gets into a situation not described in the program of its "digital brain". This means that robots cannot solve problems if the answers are not inherent in their program. A classic example of such a situation, they believe, is agreement on the agreement. In Art. 12 of the UN Convention on the use of electronic communications in international treaties refers to "electronic agents" that are designed to conclude contracts without human intervention. Practical experience shows that in the accounting system, in the end, only "ticks" are put down, while the negotiations themselves are held in any other formats. An attempt to use the robot in a complex process only complicates the process itself and the time it takes the user to solve their final task, the researchers say. However, other opinions are expressed about the possibility to teach the robot to "realize" its position in space, take into account damage, analyze experience and "plan" the near future.

Both digital and analog control systems have certain disadvantages. Analog systems have difficulty of centralized management of two or more objects. This drawback is due to the fact that it is impossible to accurately transmit analog signals over long distances. For digital systems, this is a limitation of the speed of work and the long time required for development and implementation [4]. Overcoming the identified problems on the way to creating smart robots with high artificial intelligence, these are the tasks set currently by specialists in the field of robotics.

We understand that the study of the human brain occurs using the brain of the researcher. This means that the human brain is a matter prone to self-knowledge. Can a robot have self-awareness and know itself? It seems that the answer should be negative, the robot is not a person, but only an imitation of a person who does not have consciousness and emotions. According to engineers at Columbia University (USA), in the coming years, robots will learn how to quickly adapt to unforeseen circumstances and create virtual models of themselves. Developments are also being made on the use of neural network technologies to create systems for monitoring robot functions that exceed the capabilities of standard central processing units and software.

\section{Results}

The concept of "digital robot" is absent in science, however, this term, although occasionally, is still found in the scientific literature. The definition of a digital robot can be inferred from concepts such as digital technology and a digital device. The concept of 
digital technology is inherently broader and covers not only robotics, but also other digital electronics such as slot machines, measuring instruments and other digital devices. Digital device is a technical device or device designed to receive and process information in digital form by using digital technology. Therefore, digital robot can be considered as a digital technical device designed to receive and process information in digital form using digital technologies to implement the functions laid down in it, in order to satisfy certain human needs.

The controversial question remains: can a computer or phone be classified as a robot? Most of the answers will be negative, because robot must do some work in automatic mode and, preferably, meaningfully.

A classic anthropomorphic example of such a robot can be called "Robofon", produced by the Japanese corporation Sharp. It works like a smartphone, fits easily in your pocket and, at the same time, it is a real humanoid robot. It knows how to walk and sit down, sing songs and dance, talk, choose a movie in the cinema, based on the tastes of the owner, book a table in a restaurant, take pictures and show photos using the built-in laser projector on any smooth surface. It combines the functions of a phone and a computer, like any smartphone, but at the same time it can become a secretary, interlocutor and assistant to a person.

According to O. Kharin, director of the VNIIZhT Center for Technological Information Systems, artificial intelligence is the field of computer science that is responsible for implementing various aspects of "intellectual behavior" (behavior that requires the manifestation of intelligence) using optimization algorithms, computer programs and statistics (accumulated experience or data). There are two types of artificial intelligence: "weak artificial intelligence" and "strong artificial intelligence". "Weak artificial intelligence" is a view containing a set of essentially different algorithms and methods that can solve strictly narrow-minded tasks. For example, speech recognition, recognition of objects in the image, scoring tasks, problems of diagnosis, weather forecast for tomorrow, etc. Each of these tasks requires development (training) of its own artificial intelligence algorithm. "Strong artificial intelligence" is a theory that suggests that computers can think like a person and be aware of themselves as a person. However, in practice there is still no "strong artificial intelligence" that can be aware of itself [5].

It seems that a digital robot can be called a robot that is characterized by algorithms and methods of artificial intelligence. Especially work with text, blocking algorithm on social networks, filtering and sorting algorithm used in marketing, all smart personal assistants SIRI by the automaker Chevrolet and the Siri Eyes Free application in its model Chevrolet Spark, Google voice assistants by Google, Microsoft Cortana, Alisa by Yandex, as well as the CALO cognitive assistant to military personnel (which is trained and organized). These digital devices, we think, can be attributed to the category of digital robots. Since they meet the following criteria: they are digital technical devices that perceive speech using sensors, perform functions of finding necessary information and construct a model of its further behavior in order to meet the human needs for necessary information. In addition, digital robot does not have to have signs of anthropomorphism. It can be either a real robot or a virtual one. The main thing in it is the level of intelligence. Anthropomorphism, in our opinion, should be considered from the point of view of resemblance to a person according to the intellect, not according to physical characteristics.

To systematize knowledge about robots as objects of civil law relations, a classification is proposed based on the following features:

1. In its form, robot can be both real and virtual.

A real robot is an object of material reality. Most robots have a material shell, have a moving physical structure, are equipped with an electric motor, a sensor system, a power supply and a computer "brain" that controls all the elements. Opinions are expressed about 
robots as a technogenic version of animal life and the need to endow them with the legal regime of animals in this regard.

A virtual robot is an object that does not exist as a material factual fact, but actively participates in social processes (a consultant robot, a robot bot, an assistant robot). The concept of "virtual robot" is not found in the scientific literature. However, we are familiar with such a concept as a "virtual object", which is also not legislated, but is periodically found in judicial practice, while the concept itself is not disclosed by the court.

According to P.A. Vinogradov, virtual objects are expressed externally as a certain graphic object, or as part of computer code. In this context, the author believes, difficulties may arise with the question of what is considered an object of civil law: a graphic designation of an object that an ordinary user sees, or a part of computer code that is responsible for how the ordinary user will perceive this particular object, or the combination of a computer code and a graphic component.. [6] Based on this definition, we believe that virtual robot should be considered as a single, complex and indivisible object. It does not matter what kind of graphic images virtual robot will accept (cat, dog, graphics, etc.), the main thing here is the digital code that affects the graphic device.

2. According to the technical characteristics robots can be divided into analog and digital robots.

Analog robots are robots created on the basis of analog circuits (copying biological neurons) that allow the robot to interact with the working environment (for example, BEAM robots). As a rule, analog robots exclude the use of microprocessors. The main practical application is rapid prototyping of propulsion systems, education and entertainment. Proponents of analog development believe that a large number of analog neurons can perform any task. The nature of "analog" is inherently. The digital model is a virtual creature, and digital technologies only dominate, but this path leads to a dead end.

Digital robot is a kind of digital technical device that receives and processes a huge amount of information in digital form, existing as a material or as a virtual object. There are also robots that have mixed technical characteristics, they are built on both digital and analog systems.

3. In science, it is customary to differentiate artificial intelligence according to the level of development into weak or strong artificial intelligence [7]. Modern scientific research is moving towards the development of a more developed artificial intelligence. Scientists are working on creation of such a robot, "that will be able to acquire the ability to think and be aware of itself as a separate person (in particular, to understand their own thoughts), although it is not necessary that their thought process will be similar to the human one".

In parallel, work is underway on the creation of a quantum computer that will be able to instantly solve problems the most powerful modern device spends currently years for. So, according to E. Glushkov, if in ordinary computers bits are responsible for processing information, then in quantum computers they are replaced by quantum bits (qubits). The qubit, as before, has two main values (states) that it can take: 0 and 1 . However, due to the property of quantum objects called "superposition", the qubit can take all values that are a combination of the main ones. Moreover, its quantum nature allows to be in all these states at the same time. This is the parallelism of quantum computing with qubits. To describe the exact state of the system, huge computing power and RAM are no longer needed, because to calculate a system of 100 particles, 100 qubits are needed, instead of trillions of trillions of bits. A quantum computer is still a thing of the future, notes E. Glushkov [8-9].

Despite the fact that intellectual robots exist so far only in theory, it is in relation to this category of robots that we can talk about the possibility of endowing them with special legal personality by analogy with a legal entity using legal fiction. So, according to G.A. Gadzhieva, in legal science, interpretation of the concept of "person" varies depending on the legal understanding. From the point of view of a "narrowly realistic" understanding of 
law, robot cannot be recognized as a subject of law. On the contrary, according to the approach to the legal world as a special and self-sufficient reality, the issue of legal personality of robot is a pragmatic question. As O.A. Serova notes concept of "robot", "robotics" require clear systematization, since part of such automated systems will relate to traditional objects of rights, for example, industrial robotic manipulators. However, in relation to other types of robots, whose functioning is associated with artificial intelligence and the interaction of neural networks, the question arises of possibility of their inclusion in circulation as subjects of law [10-12].

The issue of endowing a robot with legal personality is being studied from different angles: firstly, according to the subject of management - an individual or legal entity; secondly, according to the control principle - a robot with automatic control or an autonomous robot; thirdly, how the liability for damage caused by the robot is distributed. This topic always provokes lively discussions in the legal community. Most scientists criticize this issue, considering it unreasonable and untimely.

4. By type of control, robots are divided into controlled, semi-automatic and autonomous.

An autonomous robot works according to a given algorithm, based on the data received from the sensors. The following criteria of robot autonomy are distinguished in science: obtaining and independent processing of information about the environment; work in a long period of time without human intervention; ability to move any of its parts in space without human assistance; ability to avoid creating dangerous situations for a person, property or for itself - unless it is provided for by the specification, characteristics of the robot or the tasks assigned to it. Thus, an autonomous robot can independently receive information, learn, improve its algorithms and adapt to changes in the environment, but maintenance is entrusted to a person $[13,14]$. Controlled robots require the operator to control their movements. A semi-automatic robot operates according to a strictly defined program, has no sensors and is unable to adjust its actions; therefore, human participation is necessary. The 2017 Resolution of the European Parliament "Civil law on robotics" addresses such an important aspect of the use of robotics endowed with artificial intelligence as the "right to undo", the possibility of canceling an action that should become a mandatory function of the robot control system.

5. By appointment, robots are divided in industrial, service and domestic robots.

Accordingly, an industrial robot is designed to perform motor and control functions in the production process (robotic arms); service robot does useful work for people and equipment, excluding industrial automation tasks (medical robot, robot worker, PR robot, etc.); A household robot is a robot used in everyday life. This type of robots includes many completely different devices (robotic vacuum cleaner, robotic lawn mower, robot toy, personal robot and other robots that can perform various duties) used for consumer purposes.

\section{Conclusions}

The proposed classification is a systematization of the facts accumulated about robots, which is especially important because scientific theory in this area lags behind reality. For legal sciences, such a reality is public relations, where robots gradually occupy their stable place. Scientific and technological progress poses completely new questions for researchers, and each of the sciences approaches the classification of the same objects from its own special point of view.

It seems that the significance of this classification lies in the fact that it will reduce the gap between actual data and scientific theoretical constructions, and can serve as the basis for the subsequent systematization of robots and their registration system. Further scientific 
research will contribute to the resolution of certain issues of legal regulation in the field of robotics (for example, protection of personal data read by a robot from a person) and legislative consolidation of provisions on a new subject of civil relations.

\section{References}

1. N.V. Khamitov, S.V. Kiselitsa, O. L. Derkach, Probl. Soci. Robo. Philo. Psych., Sociol. 2 (10), 95-102 (2017)

2. S.I. Ozhegov, Explanatory Dictionary http://ozhegov.dicti.info/page/robot'php

3. M. G. Skvortsov, Bull. VSTU, 10 (137), 68-71 (2014)

4. S.N. Kupriyanov, D.A. Kuptsov, Cen. Prof. Manag. Acad. Busin., 87-91 (2018)

5. O. Kharin, The popularity of artificial intelligence is the result of a leap in the development of computers (2019) https://www.gudok.ru/newspaper/?ID=1455032\&archive=2019.02.28

6. P.A. Vinogradov, Yo. Sci., 15, 229-234 URL:https://moluch.ru/archive/149/42123/

7. A.N. Averkin, AI and cognitive sciences: report at the scientific conference "Artificial Intelligence Today. Problems and Prospects" (2012)

8. E. Glushkov, Just about the complex: what is a quantum computer and why is it needed https://theoryandpractice.ru/posts/13588-kvantovyy-komputer

9. O.A. Serova, Civ. La., 3, 22-24 (2018) URL: https://urfac.ru/?p=245

10. L.P. Varlamova, Zh.A. Tazhiev, Yo. Sci., 20, 121-125, 2018 URL:https://moluch.ru/archive/206/50382/ (2019).

11. N. Semeryanova, O. Fedorenko, A. Kopytova, MATEC Web of Conferences, 239, 04013 (2018) DOI: 10.1051/matecconf/201823904013

12. E. Vozniak, A.Burgundosova, A. Kopytova, MATEC Web of Conferences, 239, 01016 (2018) DOI: 10.1051/matecconf/201823901016

13. E. Vozniak, T. Slavina, A. Kopytova, MATEC Web of Conferences, 193, 04020 (2018) DOI: 10.1051/matecconf/201819304020

14. D.Izvin, , V. Lez'Er, A. Kopytova, MATEC Web of Conferences, 170, 01065 (2018) DOI: 10.1051/matecconf/201817001065

15. N. Zotkina, A. Kopytova, M. Zenkina, O. Zhigunova, MATEC Web of Conferences, 106, 08058 (2017) DOI: 10.1051/matecconf/201710608058 